\title{
Thermodynamics and Diffusion Coupling in Alloys-Application-Driven Science
}

\begin{abstract}
JOHN ÅGRN
As emphasized by Stokes (1997), the common assumption of a linear progression from basic research (science), via applied research, to technological innovations (engineering) should be questioned. In fact, society would gain much by supporting long-term research that stems from practical problems and has usefulness as a key word. Such research may be fundamental, and often, it cannot be distinguished from "basic" research if it were not for its different motivation. The development of the Calphad method and the more recent development of accompanying kinetic approaches for diffusion serve as excellent examples and are the themes of this symposium. The drivers are, e.g., the development of new materials, processes, and lifetime predictions. Many challenges of the utmost practical importance require long-term fundamental research. This presentation will address some of them, e.g., the effect of various ordering phenomena on activation barriers, and the strength and practical importance of correlation effects.
\end{abstract}

DOI: $10.1007 / \mathrm{s} 11661-011-0863-0$

(C) The Author(s) 2011. This article is published with open access at Springerlink.com

\section{INTRODUCTION-APPLIED VS BASIC RESEARCH}

IN academia, there is an unfortunate dichotomy between applied and basic research or in applicationor curiosity-driven research. One paradoxical consequence is that it is difficult to finance basic research in a field that is regarded as applied, e.g., the heat treatment of alloys. The common opinion among politicians and decision makers in organizations that fund research is that innovations follow a linear progression from basic research (science), via applied research, to technological innovations (engineering). This linear chain was questioned by Stokes ${ }^{[1]}$ in his book Pasteur's Quadrant-Basic Science and Technological Innovation. He argued that most achievements, even in basic science, during the last two centuries were actually inspired and driven by practical needs outside the scientific community. Stokes replaces the one-dimensional chain with a two-dimensional diagram (Figure 1).

It is, thus, perfectly possible that fundamental research may be driven by practical applications, i.e., societal needs. The important message to our politicians is that fundamental research may be performed in a field that is traditionally considered as applied, and it is unlikely that pure basic research will form a platform for more applied research.

In the current article, we discuss the coupling between thermodynamics and diffusion in alloys and demonstrate how the scientific research has evolved as a consequence of societal needs.

JOHN AGREN, Professor, is with the Department of Materials Science and Engineering, Royal Institute of Technology, 100444 Stockholm, Sweden. Contact e-mail: john@kth.se

This article is the 2011 Hume-Rothery Memorial Lecture.

Manuscript submitted March 10, 2011.

Article published online August 31, 2011

\section{THERMODYNAMICS AND CALPHAD}

The disastrous power of fire as well as its beneficial use has been well known throughout history. In the 18th century, craftsmen and inventors started to wonder whether fire could also be tamed to do useful work. In 1712, blacksmith master Thomas Newcomen constructed the first steam engine that made use of atmospheric pressure and heat from a fire to pump up water in English coal mines. Newcomen's steam engine was improved by James Watt in the 1780s, and the principles of "heat engines" were established during an intensive period of development to follow. That work involved, for example, the formulation of the second law (Carnot, 1824) before the first law and the entropy (Classius, 1865). The early evolution of thermodynamics as a science was thus stimulated and driven by the needs of a new and growing industry, as well as the search for the solution to engineering problems. Interesting aspects of the early theoretical development may be found in the texts by Brush $^{[2]}$ and Prigogine and Stengers. ${ }^{[3]}$ A century later, the book by Kaufman and Bernstein ${ }^{[4]}$ in 1970 inspired a new generation of researchers to apply thermodynamics and the calculation of phase equilibria to practical problems; the subsequent evolution of Calphad became connected intimately with applications in materials engineering. A thorough discussion of the development of Calphad is given in the monograph by Saunders and Miodownik. ${ }^{[5]}$

\section{DIFFUSION AND COUPLING TO THERMODYNAMICS}

Diffusion is one of the most important phenomena in nature. It has been important during the geological life of our planet, and it is important in the life of all living organisms. It plays a major role during the processing 


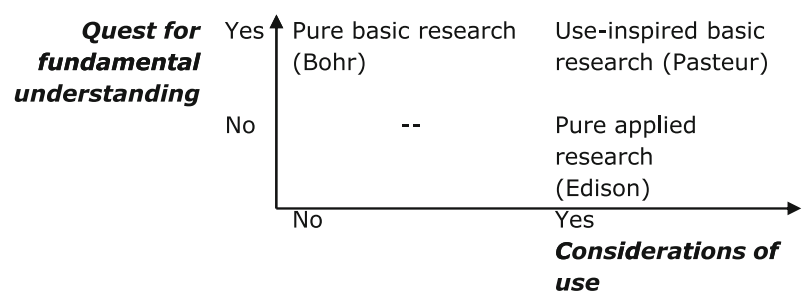

Fig. 1-Pasteur's quadrant, after Stokes ${ }^{[1]}$.

and degradation of materials. It serves as the most easyto-grasp example of an irreversible process and is thus an excellent demonstration of the second law of thermodynamics. The history of diffusion is fascinating; see, for example, the recent reviews by Barr ${ }^{[6]}$ Mehrer and Stolwijk ${ }^{[7]}$ Narasimhan, ${ }^{[8]}$ and Philibert. ${ }^{[9]}$ It is amazing to observe how the development of the field has been related to applications and practical problems. The first observations on diffusion are reported by Boyle in 1684 and were related to a color change of a metal as solid copper was soaked "into the pores of the metal." Mehl ${ }^{[10]}$ reported that Faraday and Stodart made alloys by diffusion annealing of mixed powders as early as 1820. However, usually Fick's work ${ }^{[11]}$ is taken as the starting point for the theory of diffusion. Fick was inspired by the work of Graham, ${ }^{[12]}$ who studied diffusion through membranes and actually invented the dialysis. Fick himself was active in medicine, and his main interest at that time was hydro diffusion through membranes, which he pointed out as one of the basic factors of organic life and an interesting subject as such. Unfortunately, Graham did not present any physical law that could describe his important observations, and Fick thus presented such a law in his famous paper. ${ }^{[11]}$ Before he presented the law, he had a long discussion about the molecular nature of diffusion, which did not lead to the theory but probably gave him moral support. When he finally formulates his theory, it is within a few sentences where he simply compares with Fourier's and Ohm's laws and suggests a similar law for diffusion. In the German original version of the paper, he does not even care to state the first law explicitly but moves directly to the second law. In the English abbreviated version, ${ }^{[13]}$ he states the first law in words clearly but omits the lengthy discussion of the molecular aspects.

The work in metallurgy and materials was initiated by the studies of diffusion in solid metals by RobertsAusten $^{[14]}$ who learned about diffusion when he was employed as the personal assistant to Graham, the same Graham who had inspired Fick. Roberts-Austen inspired a large number of metallurgists working on quite practical problems to look into the diffusion of alloys e.g., Mehl. ${ }^{[10]}$

Although Einstein is one of the greatest theoretical physicists ever, it should also be emphasized that he was an engineer by education and a practical person. His seminal work on the Brownian motion ${ }^{[15]}$ was actually inspired by the behavior of suspensions rather than the Brownian motion itself, which Einstein admits that he did not know much about. His analysis bridged the random molecular motion and the macroscopic deterministic behavior. Einstein's statistical treatment of diffusion evidently was an inspiration for Onsager ${ }^{[16]}$ to pursue his analysis of coupled irreversible processes, which led eventually to the reciprocity relations and a Nobel Prize. However, it is interesting that these famous relations, which were derived from rather complicated statistical considerations on the molecular behavior, in spirit similar to Fick's reasoning, could probably have been derived directly from simple assumptions. This will be discussed subsequently. Also, Onsager was interested in practical issues. For example, he suggested an apparatus for isotope separation by thermal diffusion. ${ }^{[17]}$

When Darken ${ }^{[18]}$ presented his legendary paper in 1948, which demonstrated experimentally that the gradient in chemical potential is the driving force for diffusion rather than Fick's concentration gradient, he referred to the work by Onsager and Fouss from 1932. ${ }^{[19]}$ However, it seems that he presented that idea already ${ }^{[20]}$ in a 1942 paper where he actually initially refers to industrial applications, "THE manufacture and treatment of metals comprises operations whose effectiveness depends in large measure upon diffusion phenomena." From a simple thermodynamic argument, he concluded that differences in chemical potential must be the driving force for diffusion, an idea that was strongly criticized by Mehl.

Anyhow, the introduction of the gradient in chemical potential instead of composition led to the solution of several practical problems involving joints between dissimilar materials. It may first seem strange that Fick did not draw the complete analogy between Fourier law, Ohms law, and diffusion, and he replaced temperature or electric potential with chemical potential. Instead, he chose to replace it with composition, which is not a thermodynamic potential. However, it should be realized that thermodynamics was hardly developed, e.g., the chemical potential had not been invented, when Fick published his paper. Thus, the understanding of multicomponent diffusion phenomena had to wait for almost a century.

\section{THE CHEMICAL POTENTIAL GRADIENT AS DRIVING FORCE FOR DIFFUSION -THER- MODYNAMIC COUPLING}

As mentioned, Darken ${ }^{[18]}$ demonstrated experimentally, beyond any reasonable doubt, that the gradient in chemical potential is the driving force for diffusion. Under some conditions, this could lead to "uphill" diffusion, i.e., an element may diffuse toward higher concentrations. A year earlier, he suggested that the velocity of an atom should be proportional to the force acting on the atom, i.e., the negative gradient in chemical potential. ${ }^{[21]} \mathrm{He}$ labeled the ratio between velocity and force "mobility." The flux of a component $k$ would thus be written as

$$
J_{k}=-c_{k} M_{k} \frac{\partial \mu_{k}}{\partial x}
$$

where $c_{k}$ is the concentration of $\mathrm{k}$, i.e., the number of moles per volume and $\mu_{k}$ the chemical potential of $k$. 
We have used $M$ to denote mobility rather than $B$ used by Darken. The implications of this simple relation are astonishing. Introducing the concentration gradient we recover for a binary system.

$$
J_{k}=-\left[c_{k} M_{k} \frac{\partial \mu_{k}}{\partial c_{k}}\right] \frac{\partial c_{k}}{\partial x}
$$

which is Fick's first law and the quantity inside the bracket is the diffusion coefficient. In an ideal system, we find that it is $\mathrm{R} T M_{k}, \mathrm{R}$ and $T$ being the gas constant and the temperature, respectively. The same relation between diffusion coefficient and mobility had been derived by Einstein previously, ${ }^{[22]}$ but as he only considered dilute solutions and probably did not know about the chemical potential he overlook the wide implications of the relation. So far, Eq. [2] did not tell us more than Fick's first law, except that if a solution is thermodynamically unstable, i.e., when $\partial \mu_{k} / \partial c_{k}$ is negative, the diffusion coefficient is negative and concentration differences would grow rather than level out. This is actually the starting point for the theory of spinodal decomposition. ${ }^{[23,24]}$ However, Onsager and Fouss ${ }^{[19]}$ considered electrolytes and used the mobility combined with an additional electric force. It is interesting, however, that Darken refers both to the work by Einstein and by Onsager and Fouss.

If the solution contains several components, Eq. [2] becomes

$$
J_{k}=-c_{k} M_{k} \sum_{j} \frac{\partial \mu_{k}}{\partial c_{j}} \frac{\partial c_{j}}{\partial x}=-\sum_{j} D_{k j} \frac{\partial c_{j}}{\partial x}
$$

i.e., we have to introduce several diffusion coefficients to describe the diffusion of each component. Thus, a coupling effect stems solely from the thermodynamic interactions. As an example, consider the $\mathrm{Fe}-\mathrm{C}-\mathrm{M}$ system. As a first approximation, we may assume that the mobility of carbon is unaffected of the variation in $\mathrm{M}$ content and may be evaluated from the properties of the binary $\mathrm{Fe}-\mathrm{C}$ system. Equation [3] may then be rewritten as

$$
J_{C}=-\frac{D_{C}}{\left(\partial \mu_{C} / \partial \ln c_{C}\right)} \sum_{j} \frac{\partial \mu_{C}}{\partial c_{j}} \frac{\partial c_{j}}{\partial x}
$$

where the quantity in front of the summation sign is evaluated from the binary Fe-C system. Thus we can, as a first approximation, analyze the $\mathrm{Fe}-\mathrm{C}-\mathrm{M}$ system using the thermodynamic properties of the ternary system but the diffusion data from the binary Fe-C.

This approach was taken by the current author in his Ph.D. work a long time ago, ${ }^{[25]}$ and the close agreement between calculations and experiments convinced him that this was a powerful approach of much practical importance. This was the starting point of DICTRA (Thermo-Calc Software, McMurray, PA) (Figure 2).

In general, $n^{*}(n-1)$ diffusion coefficients, but only $n$ mobilities are needed to fully describe diffusion in a system with $n$ components. These diffusion coefficients are called individual diffusion coefficients and describe diffusion relative the crystalline lattice. If we introduce

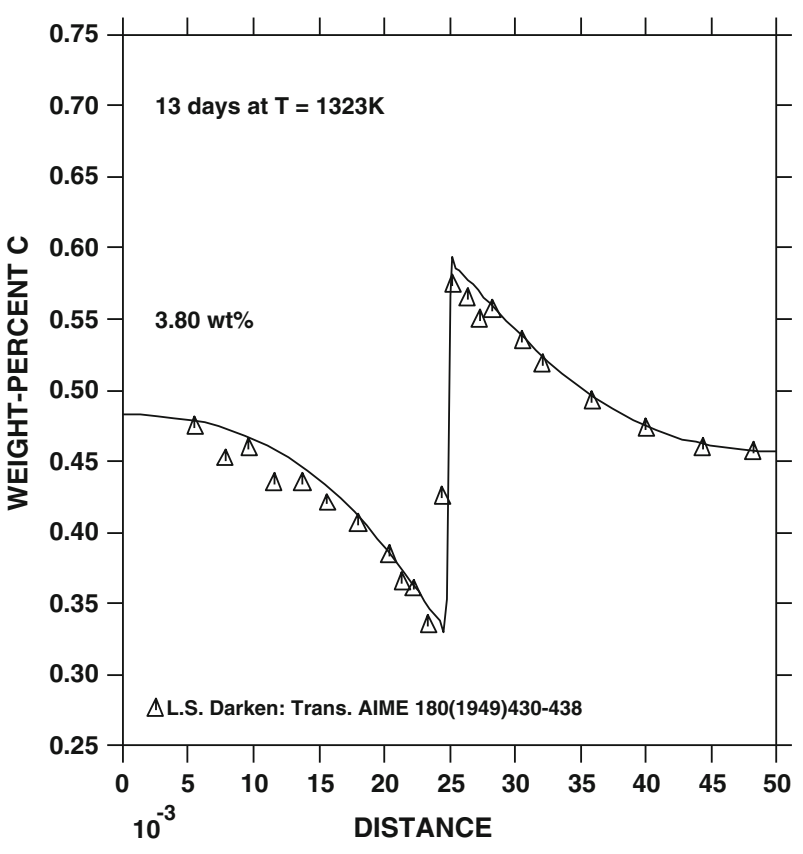

Fig. 2-DICTRA ${ }^{[26]}$ simulation of Darken's experiment, symbols from Reference 18.

the mole fraction $x_{k}$ and the molar volume $V_{m}$, we may write $c_{k} M_{k}=x_{k} M_{k} / V_{m}$ and regard this quantity in Eq. [1] as the diagonal elements in an $n^{*} n$ matrix $\mathbf{L}$, in which all off-diagonal elements vanish. Equation [1] may then be written in a condense matrix form

$$
\mathbf{J}=-\mathbf{L} \nabla \boldsymbol{\mu}
$$

where $\mathbf{J}$ is a columnar vector with elements $J_{k}$ and $\nabla \boldsymbol{\mu}$ is a columnar vector with elements $\nabla \mu_{k}$, where $\nabla$ denotes the gradient operator. The following discussion or similar ones has been presented by many authors; see, for example, the book by Kirkaldy and Young ${ }^{[27]}$ but was usually cumbersome as it made use of the notation based on vectorial and matrix elements. In the following we shall thus summarize it using the condense vectormatrix notation. Software like Matlab (MathWorks, Natick, MA) ${ }^{[28]}$ and Maple (Maplesoft, Waterloo, Ontario, Canada) ${ }^{[29]}$ easily handle matrices and vectors.

We introduce the entropy produced locally (entropy change per volume and time) and denote it with $\sigma$. From an entropy balance and the combined first and second law of thermodynamics, it is straightforward to derive an expression of $\sigma$ in terms of fluxes and forces. In the absence of temperature gradients, it is given by

$$
\sigma T=-\mathbf{J}^{T} \nabla \boldsymbol{\mu}
$$

where $\mathbf{J}^{\mathrm{T}}$ denotes the transpose of $\mathbf{J}$, i.e., $\mathbf{J}$ written as a row vector. According to the Onsager reciprocity relations, ${ }^{[16]}$ the matrix $\mathbf{L}$ is symmetric, i.e.,

$$
\mathbf{L}=\mathbf{L}^{T}
$$

In the current case, this is trivial, however, because $\mathbf{L}$ is a diagonal matrix. 


\section{FRAMES OF REFERENCE-KINETIC COUPLING}

In the previous section, we considered the $n$ independent fluxes relative the crystalline lattice, i.e., in the socalled lattice-fixed frame of reference. Often, it is more convenient to consider diffusion in other frames, e.g., interdiffusion. Transformation to any other frame of reference is achieved by multiplying $\mathbf{J}$ with a transformation matrix A that will be characteristic for the new frame of reference. The fluxes in the new frame of reference, thus, are

$$
\mathbf{J}^{\prime}=\mathbf{A} \mathbf{J}
$$

The entropy production should be independent of the choice of frame of reference and as we want to write it planes as observed in the number fixed frame is $v$, i.e., the velocity of Kirkendall markers attached to the lattice. Thus, we have

$$
\begin{gathered}
J^{\prime}=J_{2}^{\prime}=-x_{2} J_{1}+\left(1-x_{2}\right) J_{2} \\
v / V_{m}=-J=-J_{1}-J_{2}
\end{gathered}
$$

The new driving forces become

$$
\begin{gathered}
F_{1}=-\partial\left(\mu_{2}-\mu_{1}\right) / \partial x \\
F_{2}=-\left[\left(1-x_{2}\right) \partial \mu_{1} / \partial x+x_{2} \partial \mu_{2} / \partial x\right]
\end{gathered}
$$

The new $\mathbf{L}^{\prime}$ matrix becomes

$$
L^{\prime}=\frac{1}{V_{m}}\left(\begin{array}{cc}
x_{2}\left(1-x_{2}\right)\left(x_{2} M_{1}+\left(1-x_{2}\right) M_{2}\right) & x_{2}\left(1-x_{2}\right)\left(M_{1}-M_{2}\right) \\
x_{2}\left(1-x_{2}\right)\left(M_{1}-M_{2}\right) & \left(1-x_{2}\right) M_{1}+x_{2} M_{2}
\end{array}\right)
$$

in the form of Eq. [6], i.e., $\sigma T=\mathbf{J}^{\prime T} \mathbf{F}^{\prime}$, where $\mathbf{F}^{\prime}$ denotes the columnar vector containing the driving forces as elements. Clearly, they cannot generally be the gradients in the chemical potentials in other frames of reference if the entropy production shall be invariant. Thus, the forces must also be transformed, and the transformation law for the forces becomes

$$
\mathbf{F}^{\prime}=\left(\mathbf{A}^{\mathrm{T}}\right)^{-1} \mathbf{F}
$$

where $\left(\mathbf{A}^{\mathrm{T}}\right)^{-1}$ means the inverse of $\mathbf{A}^{\mathrm{T}}$. The new $\mathbf{L}^{\prime}$ matrix becomes

$$
\mathbf{L}^{\prime}=\mathbf{A L A}^{\mathrm{T}}
$$

i.e., if $\mathbf{L}$ is symmetric, then $\mathbf{L}^{\prime}$ must be symmetric. We may, thus, conclude that the validity of the Onsager relations is not affected by the change in frame of reference. It should be noted, however, that even if $\mathbf{L}$ is diagonal, $\mathbf{L}^{\prime}$ is usually not.

For the sake of simplicity, we shall demonstrate the procedure by applying it to a binary system. The number-fixed frame of reference is defined by $J_{1}^{\prime}+J_{2}^{\prime}=0$. In this frame of reference, there is only one independent elemental flux in a binary system. It is usually denoted as the interdiffusive flux. It is convenient to introduce the Kirkendall effect as a second "flux," and the transformation matrix is then defined by

$$
\mathbf{A}=\left(\begin{array}{cc}
-x_{2} & \left(1-x_{2}\right) \\
-1 & -1
\end{array}\right)
$$

The matrix A thus transforms from two independent fluxes in the lattice fixed frame to two independent fluxes in the number fixed frame. The first one is the interdiffusion flux $J^{\prime}=J_{2}^{\prime}=-J_{1}^{\prime}$ and the second one is the negative of the net-flow of atoms relative the lattice which turns out to be $v / V_{m}$. The velocity of the lattice and as can be observed, it is symmetric, i.e., the Onsager reciprocity relations are obeyed. Thus, the two fluxes are

$$
J^{\prime}=-\frac{1}{V_{m}} x_{2}\left(1-x_{2}\right)\left(x_{2} M_{1}+\left(1-x_{2}\right) M_{2}\right) \frac{\partial\left(\mu_{2}-\mu_{1}\right)}{\partial x}
$$

$$
\frac{v}{V_{m}}=-\frac{1}{V_{m}} x_{2}\left(1-x_{2}\right)\left(M_{1}-M_{2}\right) \frac{\partial\left(\mu_{2}-\mu_{1}\right)}{\partial x}
$$

where the fact that $F_{2}=0$ because of the Gibbs-Duhem relation has been used. With this transformation, the Kirkendall effect is a cross effect. By expanding the driving force $F_{1}$ in the concentration gradient, i.e.,

$$
\frac{\partial\left(\mu_{2}-\mu_{1}\right)}{\partial x}=\frac{d\left(\mu_{2}-\mu_{1}\right)}{d x_{2}} \frac{\partial x_{2}}{\partial x}
$$

we recover fully Darken's relations presented in his paper from 1948. ${ }^{[21]}$

In general, we find that a transformation from the lattice to a number-fixed frame of reference or to any other frame of reference will introduce off-diagonal elements in the $\mathbf{L}$ matrix, which however, will remain symmetric. In contrast to the thermodynamic couplings, these effects could be called kinetic coupling because they occur in the matrix of the kinetic coefficients. However, in a sense, they are only apparent and caused by the fact that we study the processes in the "wrong" frame of reference rather than the "true" frame, i.e., the lattice-fixed frame.

\section{THE ONSAGER RECIPROCITY RELATIONS}

In the previous section, we observed that the Onsager relations will be obeyed in all frames of reference if they are obeyed in one provided that the transformation rules 
are obeyed. From a mathematical point of view, the symmetric matrices containing only real numbers have an interesting property; they may be diagonalized by orthogonal matrices, which means that there is always a frame of reference where they are diagonal. The physical interpretation is that there is always a frame of reference where the different diffusion processes are independent if the $\mathbf{L}$ matrix is symmetric.

As mentioned, Onsager's proof of the reciprocity relations is complicated and difficult to follow. It is based on so-called "microscopic reversibility," ${ }^{[16]}$ which Onsager introduced inspired by the concept of detailed balance in chemistry. However, from the preceding reasoning, it is clear that the relations would follow directly if it is assumed that the processes are physically independent, i.e., there is a frame of reference where the $\mathbf{L}$ matrix is diagonal.

\section{CORRELATION EFFECTS AND OFF-DIAGONAL COEFFICIENTS}

Equation [1] may also be derived from simple absolutereaction rate arguments if it is assumed that each diffusive jump is independent of the previous jumps as well as jumps of neighboring atoms. Diffusion would thus be completely random except for some bias in the direction of lower chemical potential. However, as diffusion usually occurs by a vacancy mechanism, the probability that the atom jumps back to its previous position that is now vacant is larger than the probability that the atom would jump to another neighboring position. Such correlation effects have been discussed by several authors, e.g., Manning, ${ }^{[30]}$ and for multicomponent systems, they lead to off-diagonal mobilities, even in the lattice-fixed frame. However, it seems as they lead to a symmetric $\mathbf{L}$ matrix; see for example Dayananda. ${ }^{[31]}$ This means that there is another frame of reference where there is a diagonal $\mathbf{L}$ matrix. What is the physical significance of that "true" frame of reference? Anyhow, the magnitude of expected correlation effects is usually small compared with the scatter in experimental data, and from a practical point of view, they may be neglected in most cases.

\section{DIFFUSION IN DISPERSED SYSTEMS}

The formulation based on Eq. [1] allows us to treat redistribution of dissimilar materials. One example is a joint between a tool steel and a low-alloy steel where there is extensive redistribution of rapid interstitial solutes but much less redistribution of the sluggish substitutional solutes. The tool steel has much higher carbon content than the low-alloy steel but contains many carbide stabilizing elements that decrease the carbon chemical potential. Consequently, the carbonrich tool steel will be carburized by the carbon poor lowalloy steel, i.e., uphill diffusion. This is exactly what is observed at high temperatures where both alloys are in the one-phase austenitic state. At low temperatures, there is a complication because carbides are formed, and diffusion now occurs in a matrix with dispersed carbide particles. This problem was considered by Roper and Whittle, ${ }^{[32]}$ who mentioned high-temperature oxidation and corrosion of alloys as an important practical application. They considered a hypothetical ternary system and assumed that diffusion took place only in a matrix phase and that the fraction and composition of the two phases were given by the tie-lines in an isothermal section, i.e., thermodynamic equilibrium prevails locally. They then predicted that if a one-phase material is joined to a two-phase material, the diffusion path in the two-phase region will have a zigzag shape. The zigzag shape occurs because there is $1 \mathrm{deg}$ of freedom less in the two-phase field according to Gibbs phase rule. This approach was then used by several authors. For example, Hopfe and Morral ${ }^{[33]}$ derived analytical approximations that allowed them to consider many different cases and Engström et al. ${ }^{[34]}$ derived a

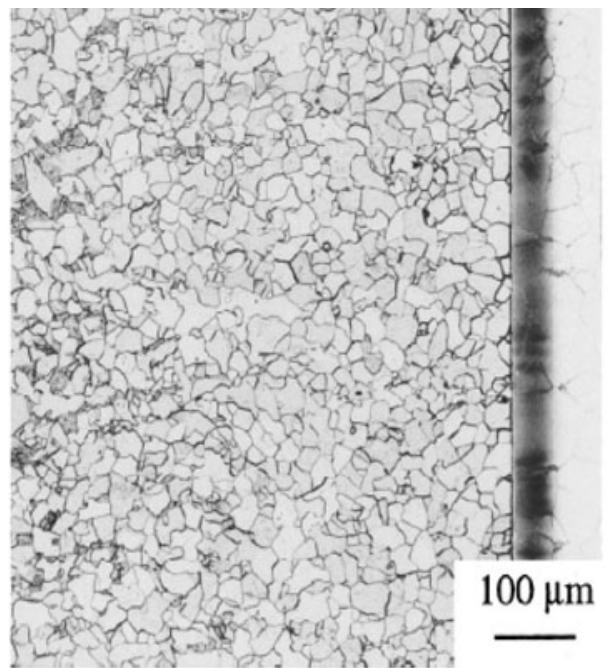

(a)

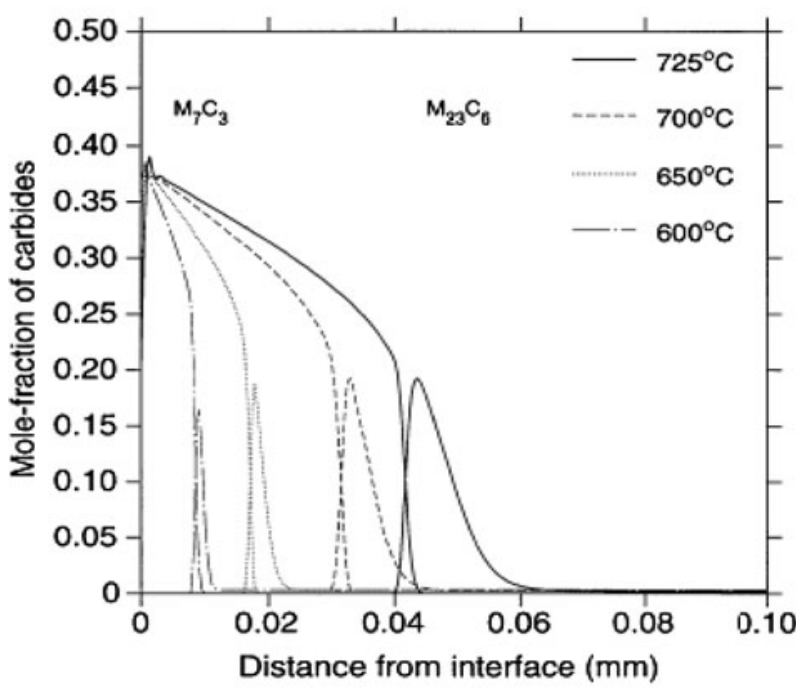

(b)

Fig. 3-(a) Microstructure of joint between low alloy steel (left) and stainless steel (right) heat treated for $100 \mathrm{~h}$ at $923 \mathrm{~K}\left(650^{\circ} \mathrm{C}\right)$. The dark-etched area on the stainless side stems from high fraction of carbides. (b) Simulated fraction of carbides on the stainless steel side at different temperatures for $10 \mathrm{~h}$. From Reference 35. 
fully numerical procedure that was implemented in DICTRA and allowed them to simulate internal carbide formation during carburizing of $\mathrm{Ni}-\mathrm{Cr}$ alloys. The latter method was applied by Helander et al. ${ }^{[35,36]}$ to joints between an austenitic stainless steel and a low alloy steel, a problem of direct industrial importance.

The predictive power of this approach could not be overestimated. The only input in addition to the temperature, alloy composition, and boundary conditions are the thermodynamic description of the Calphad type and the diffusional mobilities. No details about the microstructure are needed. In contrast, no details except the local fraction and composition of phases are obtained as output. But from a practical point of view, that output is often enough and precisely what is needed.

As an example, Figures 3(a) and (b) are reproduced from Reference 35. For 100 hours, the simulated zone of carbide precipitation in the stainless steel is approximately $80 \mu \mathrm{m}$, which agrees reasonably well with the experimental one (Figure 3(a)). For details, the reader is referred to Reference 35.



(a)

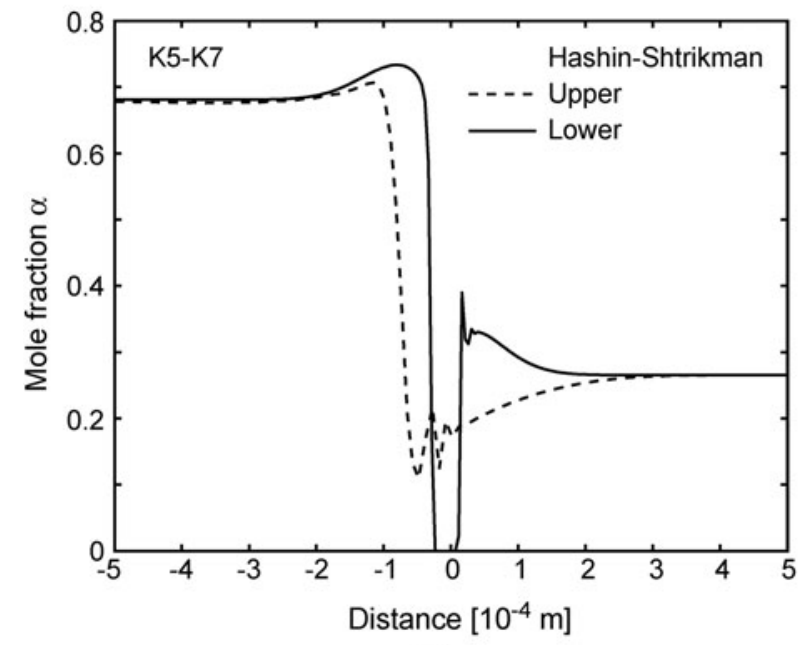

(c)
A drawback with this approach is the assumption that diffusion takes place only in the matrix phase. That excludes many practically important cases. For example, it is not possible to treat a situation where there is a change in matrix phase, e.g., if a ferritic steel is joined with an austenitic steel. The problem was solved by Larsson and Engström, ${ }^{[36]}$ who used a so-called homogenization approach to account for diffusion in all phases. Rather than considering the mobilities in a single matrix phase, the mobilities in all phases were weighed together to yield an effective mobility for an element. Figures 4(a) through (d) are taken from their work.

The experimental concentration profiles are described well by the simulation (Figure 4(d)). The variation in fraction of base-centered cubic (bcc) is predicted well using Hashin-Shtrikman lower bounds, and in particular, the thin layer of face-centered cubic (fcc) single phase is predicted with roughly the same thickness as in the experiments. Again, we conclude that a rather simple approach but based on fundamental thermodynamic

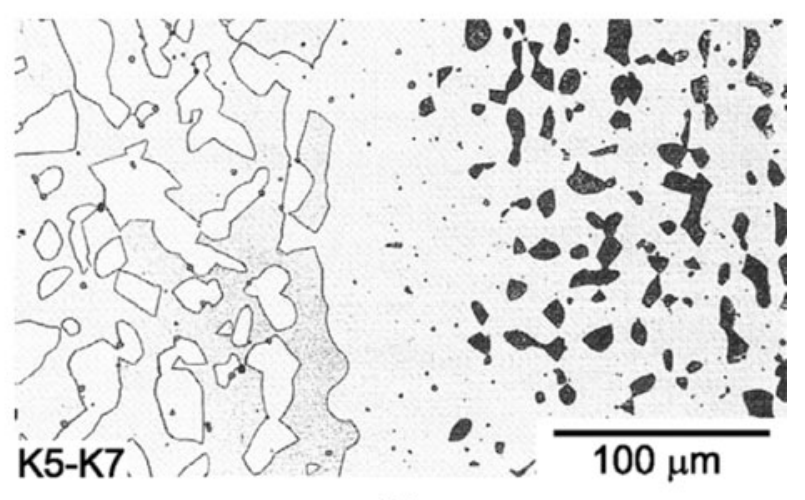

(b)

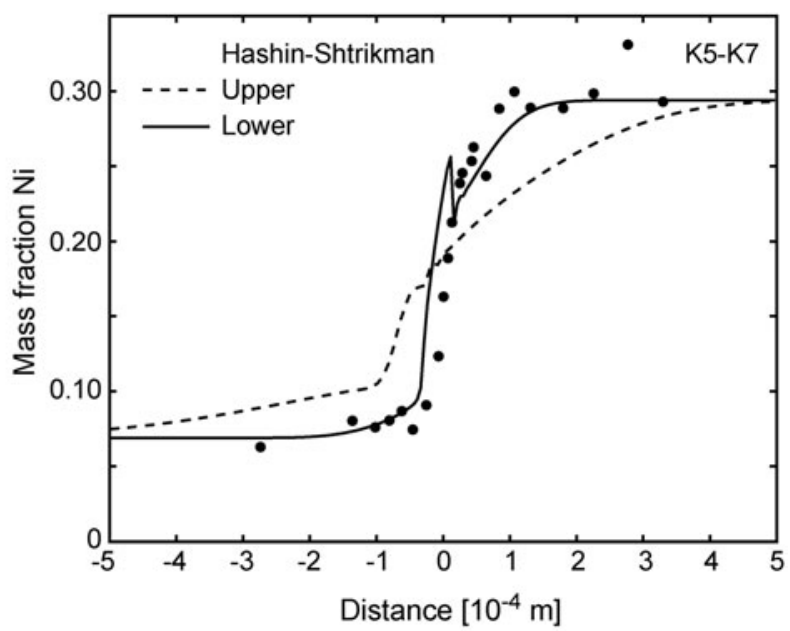

(d)

Fig. 4 - (a) $\mathrm{FCC} / \mathrm{bcc}$ diffusion couples in $\mathrm{Fe}-\mathrm{Cr}-\mathrm{Ni}$ at $1373 \mathrm{~K}\left(1100{ }^{\circ} \mathrm{C}\right) .\left(\right.$ b) Microstructure of couple $\mathrm{K} 5-\mathrm{K} 7$ heat treated at $1373 \mathrm{~K}\left(1100{ }^{\circ} \mathrm{C}\right)$ for $100 \mathrm{~h}$. (c) Simulated fraction of bec $(\alpha)$ after $100 \mathrm{~h}$ at $1373 \mathrm{~K}\left(1100^{\circ} \mathrm{C}\right)$. (d) Simulated variation in mass fraction Ni after $100 \mathrm{~h}$ at $1373 \mathrm{~K}$ $\left(1100{ }^{\circ} \mathrm{C}\right)$. From Reference 36. 
and kinetic equations predicts satisfactorily the behavior of a real alloy systems of practical importance.

\section{DIFFUSION IN TEMPERATURE GRADIENTS}

Many technical components are exposed to large temperature differences, e.g., heat-exchanger tubes and turbine blades. Such gradients may cause diffusion even if initially there are no concentration differences. This phenomenon is referred to as thermomigration. The effects may be strong, particularly for fast-diffusing interstitials. Formally, thermomigration is accounted for by adding an extra term proportional to the temperature gradient in Eq. [1]; see, for example, the work by Oriano. ${ }^{[37]}$

$$
J_{k}=-c_{k} M_{k} \frac{\partial \mu_{k}}{\partial x}-\frac{L_{k T}}{T^{2}} \frac{\partial T}{\partial x}
$$

Thus, there are off-diagonal elements in the $\mathbf{L}$ matrix even in the lattice-fixed frame of reference. The corresponding off diagonal mobility is usually given in terms of a so-called heat of transport $Q_{k}^{*}$

$$
M_{k T}=Q_{k}^{*} M_{k}
$$

where $M_{k}$ is the diffusional mobility of $k$. Various researchers evaluated experimentally the heat of transport in different systems. For example, Okafor et al. ${ }^{[38]}$ evaluated the heat of transport of carbon in $\mathrm{Fe}-\mathrm{Ni}$ alloys for high-temperature applications. Recently, Höglund and Agren ${ }^{[39]}$ implemented thermomigration in the DICTRA code. They used the experimental information from Okafor et al., evaluated the heat of transport $Q_{C}^{*}$, and simulated the experiments by Okafor et al. (see Figure 5).

Figure 5 calculated carbon profiles after 102 hours and at steady state compared with experimental measurements from Reference 38. The temperature was kept at $1400 \mathrm{~K}\left(1127^{\circ} \mathrm{C}\right)$ at the center and $1000 \mathrm{~K}\left(727^{\circ} \mathrm{C}\right)$ on both sides. Carbon segregated to the high-temperature parts.

\section{DATABASES AND EVALUATION OF MOBILITIES}

It is clear that precise data on thermodynamics and diffusion is urgently needed in many practical applications. It should also be clear from the previous sections that the most efficient way to store data is in terms of mobilities, provided that the thermodynamic properties of the system under consideration are known. A theoretical basis for such work was laid by Andersson and Agren. ${ }^{[40]}$ It has been used by several researchers since then. One may mention particularly the work on Ni-base super alloys by Campbell and colleagues ${ }^{[41,42]}$ Their database published 2002 covered $\mathrm{Ni}$ and 9 additional alloy elements, and allowed the simulation of diffusion couples of commercial alloys, e.g., Rene-N4 and Rene-N5 ${ }^{[43]}$ (Figure 6).

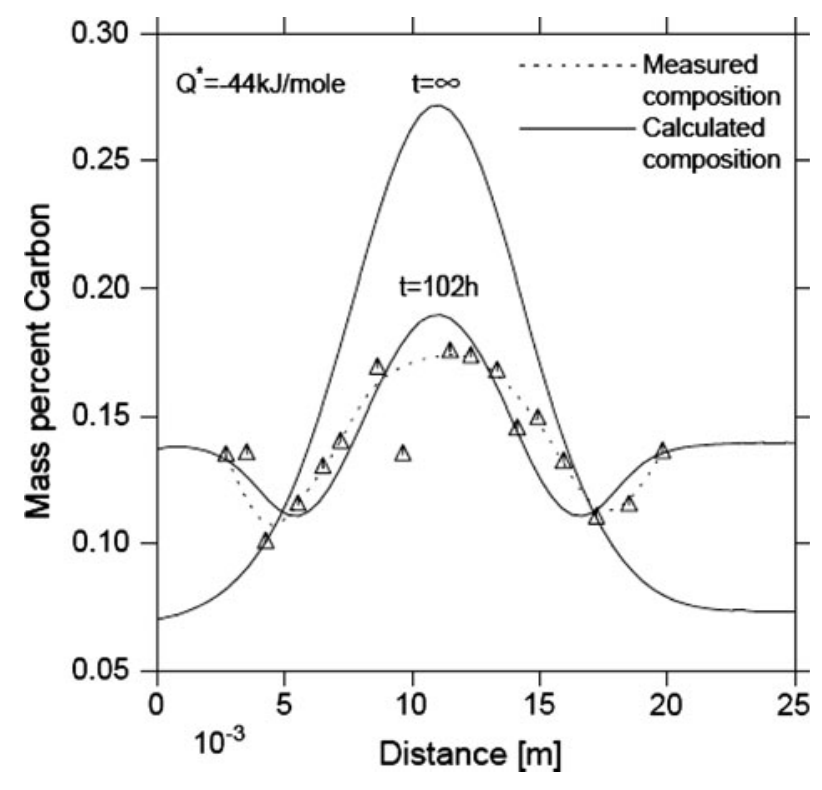

Fig. 5-Calculated carbon profiles after $102 \mathrm{~h}$ and at steady state compared with experimental measurements from Reference 38 . The temperature was kept at $1400 \mathrm{~K}\left(1127^{\circ} \mathrm{C}\right)$ at center and $1000 \mathrm{~K}$ $\left(727^{\circ} \mathrm{C}\right)$ on both sides. Carbon segregated to the high-temperature parts.

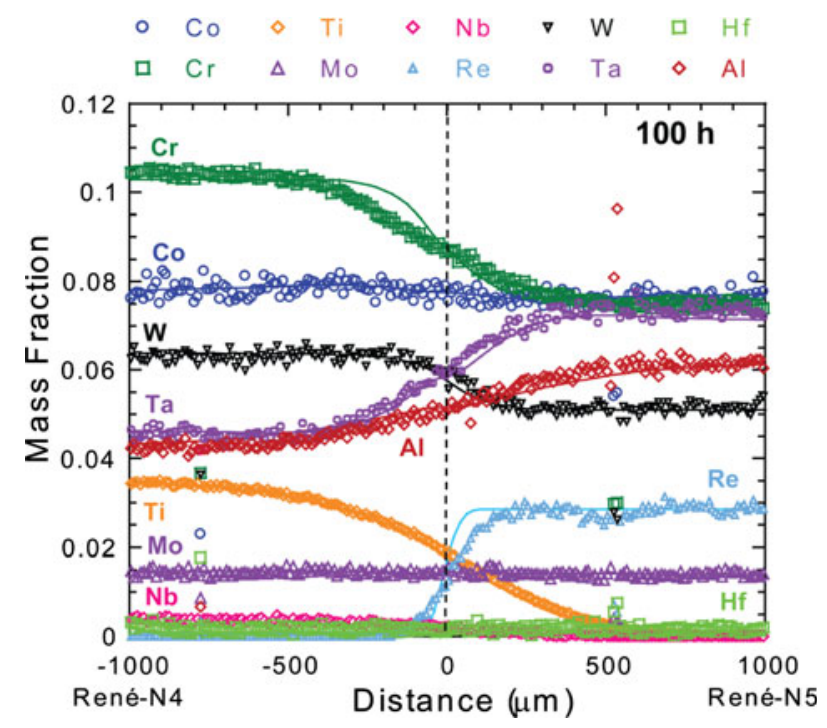

Fig. 6-Experimental and simulated concentration profiles between Rene-N4 and Rene-N5. From Reference 43.

Their database was later extended to include also the ordered phases in the $\mathrm{Ni}-\mathrm{Al}$ alloys, ${ }^{[42]}$ e.g., $\mathrm{B} 2$ and $\gamma^{\prime}$. As an example, a B2-Ni diffusion coupling was simulated for 1000 hours at $1423 \mathrm{~K}\left(1150{ }^{\circ} \mathrm{C}\right)$ (Figure 7).

The mobilities are evaluated from various pieces of experimental data in a similar way as the thermodynamic functions in the Calphad method. Such information consists of, e.g., tracer diffusion coefficients, interdiffusion coefficients, or directly measured concentration profiles. We will not discuss this in more detail, although these matters deserve their own paper. When 


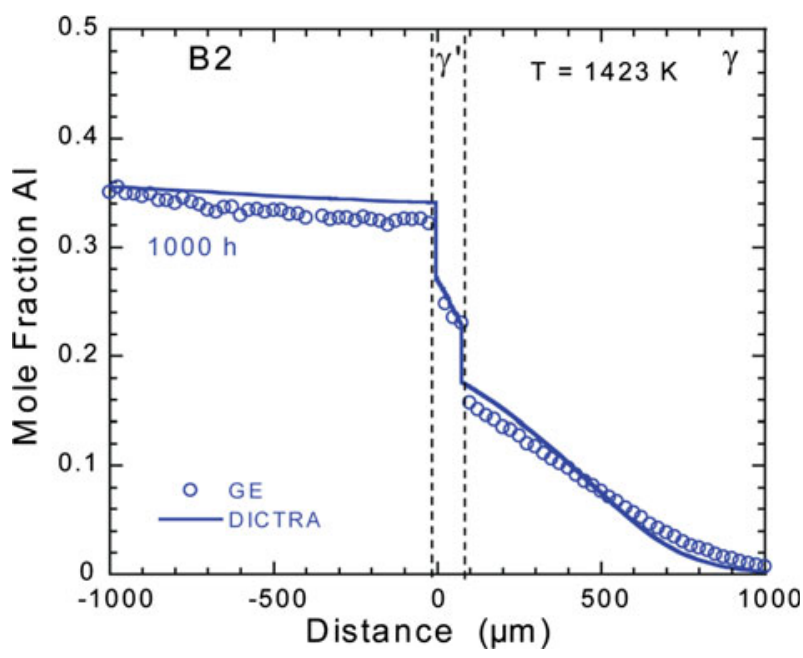

Fig. 7-Experimental and simulated concentration profiles between a B2-Ni diffusion couple kept isothermally for $1000 \mathrm{~h}$ at $1150{ }^{\circ} \mathrm{C}$ (1423 K). From Reference 42.

analyzing the experimental information, it is usually found that the different quantities depend on the composition. As it is tedious to cover those tendencies experimentally, approaches based on ab initio quantum mechanical calculations are now being tried. For example, Andersson and Simak ${ }^{[44]}$ considered the self-diffusion coefficient in $\mathrm{Cu}$. They calculated the formation energy for vacancies and divacancies as well as the activation barrier for diffusion and estimated the entropic effects by density functional theory (DFT). The authors calculated diffusivities in good agreement with experiments.

In a later paper, Andersson et al. ${ }^{[45]}$ used a similar approach to study $\mathrm{CeO}_{2}$ for application as electrolyte in solid oxide fuel cells. They managed to calculate how the activation energy for oxygen diffusion by a vacancy mechanism is affected by additions of dopants. Sandberg and Holmestad ${ }^{[46]}$ and Mantina et al. ${ }^{[47]}$ used DFT to calculate the diffusion of transition metal impurities in $\mathrm{Al}$ in reasonable agreement with experiments.

Recently, extensive research has been launched because of the renewal of nuclear power. Ferritic Fe-Cr steels have good resistance against neutron irradiation and are prime candidate materials to be used in structural parts of nuclear reactors that will be subject to extreme neutron irradiation. Sandberg et al. ${ }^{[48]}$ used ab initio methods to calculate the activation energy for carbon diffusion in bcc $\mathrm{Fe}$ and $\mathrm{Cr}$. They concluded that their results were in good agreement with experimental data.

It thus seems as information from the $a b$ initio calculations could be used as an important piece of information when evaluating mobilities.

\section{CONCLUDING REMARKS}

Already from its start, the theory of diffusion has been connected closely to practical applications. Diffusion data are needed urgently to understand and predict several important phenomena. As much experimental and theoretical work is now performed, it is important that the results are presented in a form that makes them generally useful for society.

\section{ACKNOWLEDGMENTS}

The author thanks several colleagues for much encouragement and stimulating discussions over the years: Mats Hillert, Larry Kaufman, Bo Sundman, Staffan Hertzman, Lars Höglund, Gerhard Inden, Greg Olson, Gary Purdy, Zi-Kui Liu, Anders Engström, Annika Borgenstam, Henrik Larsson, Joakim Odqvist, John Morral, Carrie Campbell, and many others.

\section{OPEN ACCESS}

This article is distributed under the terms of the Creative Commons Attribution Noncommercial License which permits any noncommercial use, distribution, and reproduction in any medium, provided the original author(s) and source are credited.

\section{REFERENCES}

1. D.E. Stokes: Pasteur's Quadrant-Basic Science and Technological Innovation, Brookings Institution Press, Washington, DC, 1997.

2. S.G. Brush: Statistical Physics and the Atomic Theory of MatterFrom Boyle and Newton to Landau and Onsager, Princeton University Press, Princeton, NJ, 1983.

3. I. Progogine and I. Stengers: Order Out of Chaos, Bantam Books, New York, NY, 1984

4. L. Kaufman and H. Bernstein: Computer Calculation of Phase Diagrams, Academic Press, Waltham, MA, 1970.

5. C.N. Saunders and A.P. Miodownik: CALPHAD Calculation of Phase Diagrams-A Comprehensive Guide, Pergamon Materials Series, Elsevier Science, Atlanta, GA, 1998.

6. L.W. Barr: Defect Diffus. Forum, 1997, vol. 143-7, pp. 3-10.

7. H. Mehrer and N.A. Stolwijk: www.diffusion-fundamentals.org, 2009.

8. T.N. Narasimhan: Physics Today, 2009, pp. 48-53.

9. J. Philibert: Diffus. Fund, 2006, vol. 4, pp. 6.1-19.

10. R.F. Mehl: Trans. AIME, 1936, vol. 122, pp. 11-56

11. A. Fick: (Poggendorff's) Annalen der Physik, 1855, vol. 170, pp. 59-86.

12. T. Graham: Diffusion Processes, Thomas Graham Symposium, Eds. J.N. Sherwood, A.V. Chadwick, W.M. Muir, and F.L. Swinton, Gordon and Breach, London, UK, 1971, vol. 1, pp. 6-7.

13. A. Fick: Phil. Mag., 1855, vols. 4, 10, no. 63, pp. 30-39.

14. W.C. Roberts-Austen: Phil. Trans. R. Soc. Lond., 1896, vol. 187, pp. 383-415.

15. A. Einstein: Ann. Phys., 1905, vol. 17, pp. 549-60.

16. L. Onsager: Phys. Rev., 1931, vol. 37, pp. 405-26.

17. A.W. Watson, L. Onsager, and A. Zucker: Rev. Sci. Instrum., 1949, vol. 20, pp. 924-27.

18. L.S. Darken: Trans. AIME, 1949, vol. 180, pp. 430-38.

19. L. Onsager and R.M. Fouss: J. Phys. Chem., 1932, vol. 36, pp. 2689-2778.

20. L.S. Darken: Trans. AIME, 1942, vol. 150, pp. 157-69.

21. L.S. Darken: Trans. AIME, 1948, vol. 175, pp. 184-94.

22. A. Einstein: Ann. Phys., 1906, vol. 19, pp. 371-81.

23. M. Hillert: Acta Metall., 1961, vol. 9, pp. 525-35.

24. J.W. Cahn: Acta Metall., 1961, vol. 9, pp. 795-801.

25. J. Ågren: Scand. J. Metall., 1982, vol. 11, pp. 3-8. 
26. A. Borgenstam, A. Engström, L. Höglund, and J. Ågren: J. Phase Equil., 2000, vol. 21, pp. 269-80.

27. J.S. Kirkaldy and D.J. Young: Diffusion in the Condensed State, Institute of Metals, London, UK, 1987.

28. http://www.mathworks.com/products/matlab/.

29. http://www.maplesoft.com/products/maple/.

30. J.R. Manning: Phys. Rev., 1961, vol. 124, pp. 470-82.

31. M.A. Dayananda: Metall. Trans., 1971, vol. 2, pp. 334-35.

32. GW Roper and DP Whittle: Met. Sci., 1981, vol. 15, pp. 148-53.

33. W.D. Hopfe and J.E. Morral: Acta Metall. Mater., 1994, vol. 42, pp. 3887-94.

34. A. Engström, L. Höglund, and J. Ågren: Metall. Mater. Trans. A, 1994, vol. 25A, pp. 1127-34.

35. T. Hellander and J. Ågren: Metall. Mater. Trans. A, 1997, vol. $28 \mathrm{~A}$, pp. $303-08$

36. H. Larsson and A. Engström: Acta Mater., 2006, vol. 54, pp. 2431-39.

37. R.A. Oriano: J. Phys. Chem. Solids, 1969, vol. 30, pp. 339-51.

38. C.I. Okafor, O.N. Carlsson, and D.M. Martin: Metall. Trans. A, 1982, vol. 13A, pp. 1713-19.
39. L. Höglund and J. Ågren: J. Phase Equil. Diffus., 2010, vol. 31, pp. $212-15$.

40. J.O. Andersson and J. Ågren: J. Appl. Phys., 1992, vol. 72, pp. $1350-55$

41. C.E. Campbell, W.J. Boettinger, and U.R. Kattner: Acta Mater., 2002, vol. 50, pp. 775-92.

42. C.E. Campbell: Acta Mater., 2008, vol. 56, pp. 4277-90.

43. U.R. Kattner and C.E. Campbell: Mater. Sci. Technol., 2009, vol. 25 , pp. $443-59$.

44. D.A. Andersson and S.I. Simak: Phys. Rev. B, 2004, vol. 70, pp. 115108-1-115108-7.

45. D.A. Andersson, S.I. Simak, N.V. Skorodumova, I.A. Abrikosov, and B. Johansson: Proc. Natl. Acad. Sci. USA, 2006, vol. 103, pp. 3518-21.

46. N. Sandberg and R. Holmestad: Phys. Rev. B, 2006, vol. 73, pp. 014108-1-014108-5.

47. M. Mantina, S.L. Shang, Y. Wang, L.Q. Chen, and Z.K. Liu: Phys. Rev. B, 2009, vol. 80, pp. 184111-1-184111-7.

48. N. Sandberg, K.O.E. Henriksson, and J. Wallenius: Phys. Rev. B, 2008, vol. 78, pp. 094110-1-094110-7. 